ALEA, Lat. Am. J. Probab. Math. Stat. 15, 151-166 (2018)

DOI: 10.30757/ALEA.v15-07

\title{
Concentration inequalities for measures of a Boolean model
}

\section{Fabian Gieringer and Günter Last}

Karlsruhe Institute of Technology, Institute of Stochastics, 76128 Karlsruhe, Germany

E-mail address: \{ fabian.gieringer, guenter.last \} @kit.edu

\begin{abstract}
We consider a Boolean model $Z$ driven by a Poisson particle process $\eta$ on a metric space $\mathbb{Y}$. We study the random variable $\rho(Z)$, where $\rho$ is a (deterministic) measure on $\mathbb{Y}$. Due to the interaction of overlapping particles, the distribution of $\rho(Z)$ cannot be described explicitly. In this note we derive concentration inequalities for $\rho(Z)$. To this end we first prove two concentration inequalities for functions of a Poisson process on a general phase space.
\end{abstract}

\section{Introduction}

Let $\mathbb{Y}$ be a locally compact separable metric space and let $\mathcal{F}$ be the space of closed subsets of $\mathbb{Y}$ equipped with a suitable $\sigma$-field. Let $\eta$ be a Poisson process on $\mathcal{F}$ with a $\sigma$-finite intensity measure $\Lambda$. If $\eta(\{K\})>0$ for some $K \in \mathcal{F}$, then we write $K \in \eta$ and say that $K$ is a particle of $\eta$. The Boolean model associated with $\eta$ is the random set $Z$ defined by

$$
Z:=\bigcup_{K \in \eta} K
$$

Let $\rho$ be a measure on $\mathbb{Y}$ satisfying

$$
\int_{\mathcal{F}} \rho(K) \Lambda(d K)<\infty
$$

Then $\rho(Z)$ is a finite random variable even though $Z$ might not be a random closed set in the sense of Matheron (1975); Schneider and Weil (2008).

The random set $Z$ is a fundamental model of stochastic geometry and continuum percolation; see Chiu et al. (2013); Matheron (1975); Schneider and Weil (2008). Explicit formulae for the distribution of geometric functionals of the Boolean model are not available, even not in the simplest case of a stationary Boolean on $\mathbb{R}^{d}$ and $\rho=\lambda_{d}(W \cap \cdot)$ being the restriction of Lebesgue measure to a convex and compact

Received by the editors March 29th, 2017; accepted January 3rd, 2018.

2010 Mathematics Subject Classification. 60D05, 60G55.

Key words and phrases. Concentration inequality, Poisson process, Boolean model, covariance identity. 
set $W$. The reason for the absence of such formulae is the interaction between the particles from $\eta$ caused by overlapping. One way out are moment formulae and central limit theorems; see e.g. Hug et al. (2016) and Last and Penrose (2017, Chapter 22). In this paper we will prove concentration inequalities of the form

$$
\mathbb{P}(F-\mathbb{E}[F] \geq r) \leq \exp \left(\inf _{s \geq 0} \int_{0}^{s} v(u) d u-s r\right), \quad r>0,
$$

where the function $v:[0, \infty) \rightarrow[0, \infty]$ is determined by $\Lambda$ and $\rho$. In the stationary Euclidean case such inequalities were first proved in Heinrich (2005). Our bounds improve these results. Moreover, we generalize the setting of Heinrich (2005) in several ways. First, we study the Boolean model on a metric space $\mathbb{Y}$ and not only on $\mathbb{R}^{d}$. Second, we will allow that compact subsets of $\mathbb{Y}$ are intersected by infinitely many Poisson particles. Hence, in general, the random set $Z$ is not closed and its boundary might have fractal properties. Roughly speaking, this means that we can allow for a $\sigma$-finite distribution of the typical grain. Closely related models of this type were introduced in Zähle (1984), a seminal paper on fractal percolation, that was almost completely ignored in the later literature. Third, we consider general measures and not only the volume. Finally, our method allows to treat also Lipschitz functions of these measures.

Similarly as in Breton et al. (2007); Houdré (2002); Houdré et al. (2008); Houdré and Privault (2002) our approach is based on a covariance identity for square integrable Poisson functionals. In fact we first prove a concentration inequality for functions of a Poisson process on a general phase space. Using the log-Sobolev inequality, related concentration inequalities were derived in Bachmann and Peccati (2016); Boucheron et al. (2003); Wu (2000).

\section{Concentration of Poisson functionals}

Let $(\mathbb{X}, \mathcal{X})$ be a measurable space and let $\Lambda$ be a $\sigma$-finite measure on $\mathbb{X}$. Let $\eta$ be a Poisson process on $\mathbb{X}$ with intensity measure $\Lambda$, defined over a probability space $(\Omega, \mathcal{A}, \mathbb{P})$; see Last and Penrose (2017). In particular, $\eta$ is a point process, that is a measurable mapping from $\Omega$ to the space $\mathbf{N}=\mathbf{N}(\mathbb{X})$ of all $\sigma$-finite measures with values in $\overline{\mathbb{N}}_{0}:=\{\infty, 0,1,2, \ldots\}$, where $\mathbf{N}$ is equipped with the smallest $\sigma$-field $\mathcal{N}$ such that $\mu \mapsto \mu(B)$ is measurable for all $B \in \mathcal{X}$. The distribution of $\eta$ is denoted by $\Pi_{\Lambda}:=\mathbb{P}(\eta \in \cdot)$. Since we are only interested in distributional properties of $\eta$, Corollary 6.5 in Last and Penrose (2017) shows that it is no restriction of generality to assume that $\eta$ is proper. This means that there exist random elements $X_{1}, X_{2}, \ldots$ in $\mathbb{X}$ such that almost surely $\eta=\sum_{n=1}^{\eta(\mathbb{X})} \delta_{X_{n}}$.

Let $0 \leq t \leq 1$ and $Y_{1}, Y_{2}, \ldots$ be a sequence of independent random variables with distribution $(1-t) \delta_{0}+t \delta_{1}$, independent of $\eta$. Define $\eta_{t}:=\sum_{n=1}^{\eta(\mathbb{X})} Y_{n} \delta_{X_{n}}$ as a $t$-thinning of $\eta$. Then $\eta_{t}$ and $\eta-\eta_{t}$ are independent Poisson processes with intensity measures $t \Lambda$ and $(1-t) \Lambda$, respectively. Given $x \in \mathbb{X}$ and a measurable function $f: \mathbf{N} \rightarrow \mathbb{R}$, the difference operator $D_{x} f$ is defined by

$$
D_{x} f(\mu):=f\left(\mu+\delta_{x}\right)-f(\mu), \quad \mu \in \mathbf{N} .
$$

We call a random variable $F$ a Poisson functional if there is a measurable $f: \mathbf{N} \rightarrow \mathbb{R}$ such that $F=f(\eta)$ almost surely. In this case we define

$$
D_{x} F:=D_{x} f(\eta), \quad x \in \mathbb{X},
$$


(which is almost surely, for $\Lambda$-almost all $x$, independent of the choice of an admissible $f)$ and further a measurable mapping $D F: \Omega \times \mathbb{X} \rightarrow \mathbb{R}$, given by $(\omega, x) \mapsto\left(D_{x} F\right)(\omega)$.

The starting point of our concentration inequalities is the following covariance identity; see Theorem 20.2 in Last and Penrose (2017). (The conditional expectation appearing in (20.2) therein can be dropped.) Earlier versions of this result can be found in Breton et al. (2007); Chen (1985); Houdré and Pérez-Abreu (1995); Houdré and Privault (2002); Privault (2009).

Proposition 2.1. Let $F=f(\eta)$ and $G=g(\eta)$ be Poisson functionals such that $F, G \in L^{2}(\mathbb{P})$ and $D F, D G \in L^{2}(\mathbb{P} \otimes \Lambda)$. Then

$$
\operatorname{Cov}(F, G)=\mathbb{E}\left[\int_{\mathbb{X}} D_{x} F \int_{0}^{1} \int_{\mathbf{N}} D_{x} g\left(\eta_{t}+\mu\right) \Pi_{(1-t) \Lambda}(d \mu) d t \Lambda(d x)\right] .
$$

For $F=f(\eta) \in L^{2}(\mathbb{P})$ we define $s_{F}:=\sup \left\{s \geq 0: e^{s F} \in L^{2}(\mathbb{P}), D e^{s F} \in\right.$ $\left.L^{2}(\mathbb{P} \otimes \Lambda)\right\}$, where the case $s_{F}=\infty$ is possible. Define

$$
V_{F}(s):=\int_{\mathbb{X}}\left(e^{s D_{x} F}-1\right) \int_{0}^{1} \int_{\mathbb{N}} D_{x} f\left(\eta_{t}+\mu\right) \Pi_{(1-t) \Lambda}(d \mu) d t \Lambda(d x), \quad 0 \leq s<s_{F} .
$$

The following bound for the cumulant generating function is the main result of this section.

Theorem 2.2. Let $F=f(\eta) \in L^{2}(\mathbb{P})$ such that $D F \in L^{2}(\mathbb{P} \otimes \Lambda)$ and let $s \in\left(0, s_{F}\right)$. Then

$$
\begin{aligned}
& \log \mathbb{E}\left[e^{s(F-\mathbb{E}[F])}\right] \\
& \leq \inf _{0<\theta<1} \frac{\theta}{1-\theta}\left(\int_{0}^{s}\left(\frac{1}{s} \log \mathbb{E}\left[e^{s V_{F}(u) / \theta}\right]-\frac{1}{u} \log \mathbb{E}\left[e^{u F}\right]\right) d u+s \mathbb{E}[F]\right) \\
& \leq \inf _{0<\theta<1} \frac{\theta}{s(1-\theta)} \int_{0}^{s} \log \mathbb{E}\left[e^{s V_{F}(u) / \theta}\right] d u .
\end{aligned}
$$

Proof: Similarly as in the proof of Corollary 6 in Houdré et al. (2008) we combine the idea of the proof of Lemma 3.2 in Houdré and Privault (2002) (see also the proof of Theorem 1 in Houdré, 2002) with Lemma 11 in Massart (2000). Let $\theta \in(0,1)$ and $s \in\left(0, s_{F}\right)$ be such that $\mathbb{E}\left[e^{s V_{F}(u) / \theta}\right]<\infty$ and let $u \in(0, s]$. Since $u<s_{F}$, we can use the covariance identity (2.1) to obtain that

$$
\begin{aligned}
\operatorname{Cov}\left(F, e^{u F}\right) & =\mathbb{E}\left[\int_{\mathbb{X}} \int_{0}^{1}\left(D_{x} e^{u F}\right) \int_{\mathbf{N}} D_{x} f\left(\eta_{t}+\mu\right) \Pi_{(1-t) \Lambda}(d \mu) d t \Lambda(d x)\right] \\
& =\mathbb{E}\left[e^{u F} V_{F}(u)\right] .
\end{aligned}
$$

Now, Lemma 11 of Massart (2000) applied to $V_{F}(u) / \theta$ and $F$ yields

$$
\frac{\mathbb{E}\left[e^{u F} V_{F}(u)\right]}{\mathbb{E}\left[e^{u F}\right]} \leq \frac{\theta \mathbb{E}\left[e^{u F} F\right]}{\mathbb{E}\left[e^{u F}\right]}+\frac{\theta}{u} \log \mathbb{E}\left[e^{u V_{F}(u) / \theta}\right]-\frac{\theta}{u} \log \mathbb{E}\left[e^{u F}\right]
$$

The combination of the last two displays leads to the inequality

$\frac{\mathbb{E}\left[F e^{u F}\right]}{\mathbb{E}\left[e^{u F}\right]}-\mathbb{E}[F]=\frac{\operatorname{Cov}\left(F, e^{u F}\right)}{\mathbb{E}\left[e^{u F}\right]} \leq \frac{\theta \mathbb{E}\left[e^{u F} F\right]}{\mathbb{E}\left[e^{u F}\right]}+\frac{\theta}{u} \log \mathbb{E}\left[e^{u V_{F}(u) / \theta}\right]-\frac{\theta}{u} \log \mathbb{E}\left[e^{u F}\right]$

and a simple rearrangement yields

$$
\frac{\mathbb{E}\left[F e^{u F}\right]}{\mathbb{E}\left[e^{u F}\right]} \leq \frac{\theta}{u(1-\theta)}\left(\frac{u}{\theta} \mathbb{E}[F]+\log \mathbb{E}\left[e^{u V_{F}(u) / \theta}\right]-\log \mathbb{E}\left[e^{u F}\right]\right) .
$$


Setting $h(t):=\log \mathbb{E}\left[e^{t F}\right]$ and $g_{u}(t):=\log \mathbb{E}\left[e^{t V_{F}(u)}\right], t \geq 0$, we have

$$
\begin{aligned}
h(s)=h(0)+\int_{0}^{s} h^{\prime}(u) d u & =\int_{0}^{s} \frac{\mathbb{E}\left[F e^{u F}\right]}{\mathbb{E}\left[e^{u F}\right]} d u \\
& \leq \int_{0}^{s} \frac{\theta}{u(1-\theta)}\left(\frac{u}{\theta} \mathbb{E}[F]+g_{u}(u / \theta)-h(u)\right) d u .
\end{aligned}
$$

By $g_{u}(0)=0$ and the convexity of $g_{u}$, we have $g_{u}\left(\frac{u}{s} t\right) \leq \frac{u}{s} g_{u}(t)$ for $t>0$, so that

$$
h(s) \leq \frac{s}{1-\theta} \mathbb{E}[F]+\frac{\theta}{1-\theta} \int_{0}^{s}\left(\frac{1}{s} g_{u}(s / \theta)-\frac{1}{u} h(u)\right) d u .
$$

From $\log \mathbb{E}\left[e^{s(F-\mathbb{E}[F])}\right]=h(s)-s \mathbb{E}[F]$ and the preceding inequality, (2.2) follows. Using Jensen's inequality, this simplifies to (2.3).

Theorem 2.2 and the well-known Chernoff bound (see Chernoff, 1952)

$$
\mathbb{P}(F-\mathbb{E}[F] \geq r) \leq \inf _{s>0} \frac{\mathbb{E}\left[e^{s(F-\mathbb{E}[F])}\right]}{e^{s r}}, \quad r \geq 0,
$$

(a direct consequence of Markov's inequality) imply a concentration inequality. If $V_{F}(\cdot)$ has a deterministic bound, we can use inequality (2.3) in (2.4) to obtain the following corollary. The (short and standard) proof is omitted.

Corollary 2.3. Let $F=f(\eta) \in L^{2}(\mathbb{P})$ such that $D F \in L^{2}(\mathbb{P} \otimes \Lambda)$. Assume that $v:\left[0, s_{F}\right) \rightarrow \mathbb{R}$ is a measurable function such that almost surely $V_{F}(s) \leq v(s)$ for each $s \in\left[0, s_{F}\right)$. Then,

$$
\mathbb{P}(F-\mathbb{E}[F] \geq r) \leq \exp \left(\int_{0}^{s} v(u) d u-s r\right), \quad s \in\left[0, s_{F}\right) .
$$

Remark 2.4. Concentration inequalities for the lower tail can be derived analogously. Under the obvious integrability assumptions, the bounds (2.2) and (2.3) hold again upon replacing $F$ by $-F$ and $V_{F}$ by $V_{-F}$. Thus, by the Chernoff bound $\mathbb{P}(F-\mathbb{E}[F] \leq-r) \leq \inf _{s>0} \mathbb{E}\left[e^{-s(F-\mathbb{E}[F])}\right] e^{-s r}, r \geq 0$, a result analogous to Corollary 2.3 gives a bound for the lower tail when $V_{-F}$ has a deterministic bound. Hence, all results relying on Corollary 2.3 can be given for the lower tail as well.

Our next result was motivated by a question in Bachmann and Peccati (2016) whether the Mecke formula (cf. Last and Penrose, 2017) can be combined with the covariance identity to yield reasonable concentration inequalities.

Theorem 2.5. Let $F=f(\eta) \in L^{2}(\mathbb{P})$ be such that $D F \in L^{2}(\mathbb{P} \otimes \Lambda)$ and such that $D F \geq 0$ holds $\mathbb{P} \otimes \Lambda$-almost everywhere. Assume further that there exist a measurable function $g: \mathbb{X} \rightarrow[0, \infty)$ and constants $a>0$ and $b \geq 0$ such that a.s. the following holds:

$$
\begin{gathered}
\int_{0}^{1} \int_{\mathbf{N}} D_{x} f\left(\eta_{t}+\mu\right) \Pi_{(1-t) \Lambda}(d \mu) d t \leq g(x), \quad \Lambda \text {-a.e. } x \in \mathbb{X} \\
\int D_{x} f\left(\eta-\delta_{x}\right) g(x) \eta(d x) \leq a F+b .
\end{gathered}
$$

Then

$$
\begin{aligned}
& \mathbb{P}(F-\mathbb{E}[F] \geq r) \\
& \leq \exp \left(\inf _{0<s<a^{-1} \wedge s_{F}}\left(-\frac{b s}{a}-s \mathbb{E}[F]-\left(\frac{\mathbb{E}[F]}{a}+\frac{b}{a^{2}}\right) \log (1-a s)-s r\right)\right) .
\end{aligned}
$$


In particular, if $a^{-1} \leq s_{F}$, we have

$$
\mathbb{P}(F-\mathbb{E}[F] \geq r) \leq \exp \left(-\frac{1}{a}\left[r+\left(\mathbb{E}[F]+\frac{b}{a}\right) \log \left(\frac{\mathbb{E}[F]+b / a}{r+\mathbb{E}[F]+b / a}\right)\right]\right) .
$$

Proof: Let $0<u \leq s<a^{-1} \wedge s_{F}$. By the covariance identity (2.1) and assumption (2.6), we have

$$
\operatorname{Cov}\left(F, e^{u F}\right) \leq \mathbb{E}\left[\int D_{x} e^{u F} g(x) \Lambda(d x)\right] .
$$

Applying the Mecke formula and the elementary bound $e^{z}-1 \leq z e^{z}, z \in \mathbb{R}$, yields

$$
\begin{aligned}
\operatorname{Cov}\left(F, e^{u F}\right) & \leq \mathbb{E}\left[\int D_{x} e^{u f\left(\eta-\delta_{x}\right)} g(x) \eta(d x)\right] \\
& =\mathbb{E}\left[\int e^{u f\left(\eta-\delta_{x}\right)}\left(e^{u D_{x} f\left(\eta-\delta_{x}\right)}-1\right) g(x) \eta(d x)\right] \\
& \leq u \mathbb{E}\left[\int e^{u f(\eta)} D_{x} f\left(\eta-\delta_{x}\right) g(x) \eta(d x)\right] .
\end{aligned}
$$

Assumption (2.7) yields that $\operatorname{Cov}\left(F, e^{u F}\right) \leq u a \mathbb{E}\left[F e^{u F}\right]+u b \mathbb{E}\left[e^{u F}\right]$. It follows that

$$
\frac{\mathbb{E}\left[F e^{u F}\right]}{\mathbb{E}\left[e^{u F}\right]} \leq \frac{\mathbb{E}[F]+u b}{1-a u} .
$$

Setting $h(u):=\log \mathbb{E}\left[e^{u F}\right]$, we get $h^{\prime}(u) \leq(\mathbb{E}[F]+u b) /(1-a u)$ and hence, by integration and $h(0)=0$, the bound $\log \mathbb{E}\left[e^{s F}\right] \leq-b s / a-\left(\mathbb{E}[F] / a+b / a^{2}\right) \log (1-$ $a s)$. Using the Chernoff bound (2.4), we obtain (2.8). Inequality (2.9) follows by optimising with $s=r /(a r+a \mathbb{E}[F]+b)$. This choice of $s$ is at most $a^{-1}$, since $F+b / a \geq 0$ a.s. by assumption (2.7), and the case $\mathbb{E}[F]+b / a=0$ can be ruled out as this implies $D F \equiv 0$ and therefore a trivial concentration.

\section{General Boolean models}

In this section we shall consider a Poisson process on a suitable class $\mathcal{F}^{\prime}$ of closed subsets of a locally compact separable Hausdorff space $\mathbb{Y}$. Let $\mathcal{F} \equiv \mathcal{F}(\mathbb{Y})$ denote the class of all closed subsets of $\mathbb{Y}$ equipped with the Fell topology; see Matheron (1975); Schneider and Weil (2008). The associated Borel $\sigma$-field is denoted by $\mathcal{B}(\mathcal{F})$. Let $\rho$ be a measure on $(\mathbb{Y}, \mathcal{Y})$, where $\mathcal{Y}$ denotes the Borel $\sigma$-field of $\mathbb{Y}$. Let $\mathcal{F}^{\prime} \in \mathcal{B}(\mathcal{F})$ be such that $\rho$ is finite on $\mathcal{F}^{\prime}$ and $K \mapsto \rho(K)$ is measurable on $\mathcal{F}^{\prime}$. We assume that $\mathcal{F}^{\prime}$ is closed under finite unions and equip $\mathcal{F}^{\prime}$ with the trace $\sigma$-field $\mathcal{B}\left(\mathcal{F}^{\prime}\right):=\left\{B \cap \mathcal{F}^{\prime}: B \in \mathcal{B}(\mathcal{F})\right\}$.

Let $\eta$ be a Poisson process on $\mathcal{F}^{\prime}$ whose intensity measure $\Lambda$ satisfies

$$
\int_{\mathcal{F}^{\prime}} \rho(K) \Lambda(d K)<\infty
$$

Let $B_{n} \in \mathcal{B}\left(\mathcal{F}^{\prime}\right), n \in \mathbb{N}$, be such that $B_{n} \uparrow \mathcal{F}^{\prime}$ and $\Lambda\left(B_{n}\right)<\infty$ for all $n \in \mathbb{N}$. Let $\mathbf{N}_{l}$ denote the measurable set of all $\mu \in \mathbf{N} \equiv \mathbf{N}\left(\mathcal{F}^{\prime}\right)$ satisfying $\mu\left(B_{n}\right)<\infty$ for each $n \in \mathbb{N}$. Note that $\mathbb{P}\left(\eta \in \mathbf{N}_{l}\right)=\Pi_{\Lambda}\left(\mathbf{N}_{l}\right)=1$. Define

$$
Z(\mu):=\bigcup_{\mu(\{K\})>0} K, \quad \mu \in \mathbf{N}_{l}
$$


and $Z(\mu):=\emptyset$ for $\mu \in \mathbf{N} \backslash \mathbf{N}_{l}$. The random set $Z:=Z(\eta)$ is called the Boolean model governed by $\eta$. Now consider the function $f: \mathbf{N} \rightarrow[0, \infty)$ given by

$$
f(\mu):=\mathbf{1}\{\rho(Z(\mu))<\infty\} \rho(Z(\mu)), \quad \mu \in \mathbf{N},
$$

with the convention $0 \cdot \infty:=0$. Our goal is to obtain a concentration inequality for $F:=f(\eta)$.

Campbell's formula (Proposition 2.7 in Last and Penrose, 2017) and assumption (3.1) show that $\int \rho d \eta<\infty$ a.s., so that the sub-additivity of $\rho$ shows that $\rho(Z)<\infty$ a.s. In particular $\mathbb{P}(F=\rho(Z))=1$. However, we need to check that $\rho(Z)$ is a random variable. This follows from the assumption on $\rho$ and the next lemma.

Lemma 3.1. The mapping $(x, \mu) \mapsto \mathbf{1}\{x \in Z(\mu)\}$ is measurable on $\mathbb{Y} \times \mathbf{N}$. Furthermore, for each $K \in \mathcal{Y}$ with $\rho(K)<\infty$, the mapping $\mu \mapsto \rho(Z(\mu) \cap K)$ is measurable on $\mathbf{N}$. Finally $\mu \mapsto \rho(Z(\mu))$ is measurable on $\mathbf{N}$.

Proof: By Theorem 1-2-1 in Matheron (1975), $\mathcal{F}$ is a compact and separable Hausdorff space and hence $\mathcal{F}^{\prime}$ (equipped with the trace $\sigma$-field) is a Borel space; see Kallenberg (2002, Theorem A1.1). By Last and Penrose (2017, Proposition 6.2) (see also the proof of Last and Penrose, 2017, Proposition 6.3), there exist measurable functions $\pi_{n}: \mathbf{N} \rightarrow \mathcal{F}^{\prime}$ such that

$$
\mu=\sum_{n=1}^{\mu\left(\mathcal{F}^{\prime}\right)} \delta_{\pi_{n}(\mu)}, \quad \mu \in \mathbf{N}_{l}
$$

This shows that $Z(\mu)=\cup_{n=1}^{\mu\left(\mathcal{F}^{\prime}\right)} \pi_{n}(\mu)$ for each $\mu \in \mathbf{N}_{l}$. By Matheron (1975, Theorem 2-5-1), the mapping $(K, x) \mapsto \mathbf{1}\{x \in K\}$ is measurable on $\mathcal{F} \times \mathbb{Y}$. Since

$$
\mathbf{1}\{x \notin Z(\mu)\}=\prod_{n=1}^{\mu\left(\mathcal{F}^{\prime}\right)} \mathbf{1}\left\{x \notin \pi_{n}(\mu)\right\}, \quad \mu \in \mathbf{N}_{l},
$$

this proves the first assertion. The second assertion follows from Fubini's theorem.

By monotone convergence, the third assertion follows, once we have shown that

$$
\mu \mapsto \rho\left(\bigcup_{m=1}^{\min \left\{\mu\left(\mathcal{F}^{\prime}\right), n\right\}} \pi_{m}(\mu)\right)
$$

is measurable for each $n \in \mathbb{N}$. By Matheron (1975, Corollary 1-2-1) the mapping $\left(L, L^{\prime}\right) \mapsto L \cup L^{\prime}$ is measurable on $\mathcal{F} \times \mathcal{F}$ and hence also on $\mathcal{F}^{\prime} \times \mathcal{F}^{\prime}$. Since $\mathcal{F}^{\prime}$ is closed under unions, it follows that $\mu \mapsto \bigcup_{m=1}^{\min \left\{\mu\left(\mathcal{F}^{\prime}\right), n\right\}} \pi_{m}(\mu)$ is a measurable mapping from $\mathbf{N}_{l}$ to $\mathcal{F}^{\prime}$. Since $L \mapsto \rho(L)$ is measurable on $\mathcal{F}^{\prime}$, the final assertion now follows.

Let $t \in[0,1]$. We now compute the probability that a point $y \in \mathbb{Y}$ lies inside the Boolean model with intensity measure $t \Lambda$. We use the notation

$$
\mathcal{F}_{y}^{\prime}:=\left\{K \in \mathcal{F}^{\prime}: y \in K\right\}, \quad y \in \mathbb{Y} .
$$

Since $(K, y) \mapsto \mathbf{1}\{y \in K\}$ is $\mathcal{B}(\mathcal{F}) \otimes \mathcal{Y}$-measurable, this is a measurable set. The first definition in (3.2) and the defining properties of a Poisson process yield

$$
\int \mathbf{1}\{y \in Z(\mu)\} \Pi_{t \Lambda}(d \mu)=1-\mathbb{P}\left(\eta_{t}\left(\mathcal{F}_{y}^{\prime}\right)=0\right)=1-\exp \left(-t \Lambda\left(\mathcal{F}_{y}^{\prime}\right)\right) .
$$


Lemma 3.2. We have that

$$
D_{K} f(\mu)=\rho(K)-\rho(Z(\mu) \cap K), \quad \Pi_{\Lambda} \otimes \Lambda \text {-a.e. }(\mu, K) .
$$

Proof: For each $\mu \in \mathbf{N}_{l}$ and each $K \in \mathcal{F}^{\prime}$ we have that

$$
\left.D_{K} f(\mu)=\rho\left(Z\left(\mu+\delta_{K}\right)\right)-\rho(Z(\mu))=\rho(Z(\mu) \cup K)\right)-\rho(Z(\mu)) .
$$

Since $\Pi_{\Lambda}\left(\left\{\mu \in \mathbf{N}_{l}: \rho(Z(\mu))<\infty\right\}\right)=1$ we can use the additivity of $\rho$ to conclude the proof.

Lemma 3.3. Let $t \in[0,1]$ and $K \in \mathcal{F}^{\prime}$. Then $\mathbb{P}$-a.s.

$$
\int D_{K} f\left(\eta_{t}+\mu\right) \Pi_{(1-t) \Lambda}(d \mu) \leq \int \mathbf{1}\{y \in K\} \exp \left(-(1-t) \Lambda\left(\mathcal{F}_{y}^{\prime}\right)\right) \rho(d y) .
$$

Proof: By Lemma 3.2 and the superposition theorem for Poisson processes we have a.s.

$$
D_{K} f\left(\eta_{t}+\mu\right) \leq \rho(K)-\rho(Z(\mu) \cap K), \quad \Pi_{(1-t) \Lambda} \otimes \Lambda \text {-a.e. }(\mu, K) .
$$

Furthermore,

$$
\begin{aligned}
\int \rho(Z(\mu) \cap K) \Pi_{(1-t) \Lambda}(d \mu) & =\iint 1\{y \in Z(\mu)\} 1\{y \in K\} \Pi_{(1-t) \Lambda}(d \mu) \rho(d y) \\
& =\rho(K)-\int 1\{y \in K\} \exp \left(-(1-t) \Lambda\left(\mathcal{F}_{y}^{\prime}\right)\right) \rho(d y),
\end{aligned}
$$

where we have used (3.4). The assertion now follows.

In what follows we shall use the full measure properties of $\rho$. Define

$$
\rho^{*}(K):=\int_{K} \tau\left(\Lambda\left(\mathcal{F}_{y}^{\prime}\right)\right) \rho(d y), \quad K \in \mathcal{F}^{\prime},
$$

where $\tau:[0, \infty] \rightarrow[0,1]$ is given by $\tau(z):=\left(1-e^{-z}\right) / z$ for $z \in(0, \infty), \tau(0):=$ $\lim _{z \downarrow 0} \tau(z)=1$ and $\tau(\infty):=\lim _{z \rightarrow \infty} \tau(z)=0$. We define a measure $\nu^{*}$ on $[0, \infty)$ by

$$
\nu^{*}:=\int_{\mathcal{F}^{\prime}} \mathbf{1}\{\rho(K) \in \cdot\} \frac{\rho^{*}(K)}{\rho(K)} \Lambda(d K),
$$

with the convention $0 / 0:=0$ and another measure $\nu$ on $[0, \infty)$ by

$$
\nu:=\int_{\mathcal{F}^{\prime}} \mathbf{1}\{\rho(K)>0, \rho(K) \in \cdot\} \Lambda(d K) .
$$

Naturally, our concentration inequalities require the constant

$$
s_{0}:=\sup \left\{s>0: \int_{\mathcal{F}^{\prime}} \mathbf{1}\{\rho(K)>1\} e^{s \rho(K)} \Lambda(d K)<\infty\right\}
$$

to be positive.

Define a function $\phi:[0, \infty) \rightarrow \mathbb{R}, \phi(z):=e^{z}-1-z$.

Theorem 3.4. Assume that (3.1) holds and that $s_{0}>0$, where $s_{0}$ is given by (3.6). Then

$$
\mathbb{P}(\rho(Z)-\mathbb{E}[\rho(Z)] \geq r) \leq \exp \left(\inf _{0<s<s_{0}}\left(\int \phi(s u) \nu^{*}(d u)-s r\right)\right), \quad r>0 .
$$


Proof: We write $\eta=\sum_{k=1}^{\eta\left(\mathcal{F}^{\prime}\right)} \delta_{Z_{k}}$. Let $n \in \mathbb{N}$. Then $\eta_{n}:=\sum_{k=1}^{\eta\left(\mathcal{F}^{\prime}\right)} \mathbf{1}\left\{\rho\left(Z_{k}\right) \leq n\right\} \delta_{Z_{k}}$ is a Poisson process with intensity measure $\Lambda_{n}(d K):=\mathbf{1}\{\rho(K) \leq n\} \Lambda(d K)$. Define $Z_{n}:=Z\left(\eta_{n}\right)$ and $F_{n}:=\rho\left(Z_{n}\right)$. We wish to apply Corollary 2.3 to the pair $\left(\eta_{n}, F_{n}\right)$. We start by checking the integrability properties of the Poisson functional $F_{n}$. First, we obtain from Lemma 3.2 that

$$
\mathbb{E}\left[\int\left(D_{K} F_{n}\right)^{2} \Lambda_{n}(d K)\right] \leq \int \mathbf{1}\{u \leq n\} u^{2} \nu(d u) \leq n \int u \nu(d u)
$$

which is finite by (3.1). Since $\mathbb{E}\left[F_{n}\right]<\infty$ the Poincaré inequality (see Last and Penrose, 2017, Corollary 18.8) shows that $\mathbb{E}\left[F_{n}^{2}\right]<\infty$. Secondly, we have for each $s \geq 0$ that

$$
\mathbb{E}\left[e^{2 s F_{n}}\right] \leq \mathbb{E}\left[\exp \left[2 s \int \rho(K) \eta_{n}(d K)\right]\right]=\exp \left[\int\left(e^{2 s \rho(K)}-1\right) \Lambda_{n}(d K)\right],
$$

where we have used a well-known formula for Poisson processes (see e.g. Last and Penrose, 2017). The integral in the exponent is dominated by a multiple (depending on $n$ and $s)$ of $\int \mathbf{1}\{u \leq n\} u \nu(d u)$ and hence finite. Thirdly, we have

$$
\begin{aligned}
\mathbb{E}\left[\int\left(D_{K} e^{s F_{n}}\right)^{2} \Lambda_{n}(d K)\right] & =\int \mathbb{E}\left[\left(e^{s F_{n}}\left(e^{s D_{K} F_{n}}-1\right)\right)^{2}\right] \Lambda_{n}(d K) \\
& \leq \mathbb{E}\left[e^{2 s F_{n}}\right] \int\left(e^{s \rho(K)}-1\right)^{2} \Lambda_{n}(d K) .
\end{aligned}
$$

This is finite, since $\int \mathbf{1}\{u \leq n\}\left(e^{s u}-1\right)^{2} \nu(d u)$ is bounded by a multiple of $\int u \nu(d u)$.

By Lemma 3.2 and Lemma 3.3 (applied with $\left(\eta_{n}, F_{n}\right)$ in place of $(\eta, F)$ ),

$$
\begin{aligned}
V_{F_{n}}(s) & \leq \int_{\mathcal{F}^{\prime}}\left(e^{s \rho(K)}-1\right) \int_{\mathbb{Y}} \mathbf{1}\{y \in K\} \int_{0}^{1} \exp \left(-(1-t) \Lambda_{n}\left(\mathcal{F}_{y}^{\prime}\right)\right) d t \rho(d y) \Lambda_{n}(d K) \\
& =\int_{\mathcal{F}^{\prime}}\left(e^{s \rho(K)}-1\right) \int_{K} \tau\left(\Lambda_{n}\left(\mathcal{F}_{y}^{\prime}\right)\right) \rho(d y) \Lambda_{n}(d K)=: h_{n}(s) .
\end{aligned}
$$

Let $\rho_{n}^{*}(K):=\int_{K} \tau\left(\Lambda_{n}\left(\mathcal{F}_{y}^{\prime}\right)\right) \rho(d y)$. Then we have

$$
\begin{aligned}
\int_{0}^{s} h_{n}(u) d u & =\int_{\mathcal{F}^{\prime}} \mathbf{1}\{\rho(K)>0\} \rho_{n}^{*}(K) \int_{0}^{s}\left(e^{u \rho(K)}-1\right) d u \Lambda_{n}(d K) \\
& =\int \mathbf{1}\{\rho(K)>0\} \rho_{n}^{*}(K)\left(\frac{e^{s \rho(K)}-1}{\rho(K)}-s\right) \Lambda_{n}(d K) \\
& =\int \frac{\rho_{n}^{*}(K)}{\rho(K)} \phi(s \rho(K)) \Lambda_{n}(d K) .
\end{aligned}
$$

For each $r>0$ we now obtain from (2.5) that

$$
\mathbb{P}\left(F_{n}-\mathbb{E}\left[F_{n}\right] \geq r\right) \leq \exp \left(\int \mathbf{1}\{\rho(K) \leq n\} \frac{\rho_{n}^{*}(K)}{\rho(K)} \phi(s \rho(K)) \Lambda(d K)-s r\right) .
$$

As $n \rightarrow \infty$ we have $Z_{n} \uparrow Z$ and hence $F_{n} \uparrow \rho(Z)$. Monotone convergence implies $\mathbb{E}\left[F_{n}\right] \rightarrow \mathbb{E}[\rho(Z)]$. We now assume that $s \in\left(0, s_{0}\right)$ and assert that

$$
\lim _{n \rightarrow \infty} \int \mathbf{1}\{\rho(K) \leq n\} \frac{\rho_{n}^{*}(K)}{\rho(K)} \phi(s \rho(K)) \Lambda(d K)=\int \frac{\rho^{*}(K)}{\rho(K)} \phi(s \rho(K)) \Lambda(d K) .
$$

Indeed, we have for each $y \in \mathbb{Y}$ that $\lim _{n \rightarrow \infty} \Lambda_{n}\left(\mathcal{F}_{y}^{\prime}\right)=\Lambda\left(\mathcal{F}_{y}^{\prime}\right)$, and since $\tau(\cdot) \leq 1$ we obtain for each $K \in \mathcal{F}^{\prime}$ from dominated convergence that $\lim _{n \rightarrow \infty} \rho_{n}^{*}(K)=$ 
$\rho^{*}(K)$. Hence (3.10) follows from dominated convergence once we have shown that $\int \phi(s \rho(K)) \Lambda(d K)$ is finite. By assumption (3.6) it is sufficient to show that

$$
\int 1\{u \leq 1\} \phi(s u) \nu(d u)<\infty .
$$

For $u \in[0,1]$ the definition of $\phi$ implies that $\phi(s u) \leq u \phi(s)$ and (3.11) follows. Let $\varepsilon>0$ such that $r-\varepsilon>0$. Fatou's Lemma shows that

$$
\begin{aligned}
\mathbb{P}(\rho(Z)-\mathbb{E}[\rho(Z)]>r-\varepsilon) & \leq \liminf _{n \rightarrow \infty} \mathbb{P}\left(F_{n}-\mathbb{E}\left[F_{n}\right]>r-\varepsilon\right) \\
& \leq \exp \left(\int \frac{\rho^{*}(K)}{\rho(K)} \phi(s \rho(K)) \Lambda(d K)-s(r-\varepsilon)\right),
\end{aligned}
$$

where we have used (3.10) and (3.9) to obtain the second inequality. Letting $\varepsilon \rightarrow 0$, we obtain the asserted concentration inequality (3.7).

Remark 3.5. Theorem 3.4 can be extended to a Poisson functional $G:=T(\rho(Z))$, where $T:[0, \infty) \rightarrow \mathbb{R}$ is Lipschitz with Lipschitz constant $c_{T} \geq 0$. We then have

$$
\mathbb{P}(G-\mathbb{E}[G] \geq r) \leq \exp \left(\inf _{0<s<s_{0} / c_{T}}\left(\int \phi\left(c_{T} s u\right) \nu^{*}(d u)-s r\right)\right), \quad r>0 .
$$

Define a function $h:[0, \infty) \rightarrow[0, \infty]$ by

$$
h(s):=\int_{0}^{\infty} u\left(e^{s u}-1\right) \nu^{*}(d u), \quad s \geq 0 .
$$

If $\nu^{*}=0$, then $h \equiv 0$. Otherwise $h$ is finite and strictly increasing on $\left[0, s_{0}\right)$. Let $h^{-1}:[0, \infty) \rightarrow[0, \infty]$ denote the generalized inverse of $h$, defined by

$$
h^{-1}(u):=\inf \{s \geq 0: h(s) \geq u\}, \quad u \geq 0,
$$

where $\inf \emptyset:=\infty$. If $\nu^{*}=0$, then $h^{-1} \equiv \infty$ on $(0, \infty)$. Otherwise $h^{-1}$ is strictly increasing and continuous on $\left[0, h\left(s_{0}-\right)\right)$, where $h\left(s_{0}-\right):=\lim _{s \uparrow s_{0}} h(s)$.

Theorem 3.6. Under the assumptions of Theorem 3.4,

$$
\mathbb{P}(\rho(Z)-\mathbb{E}[\rho(Z)] \geq r) \leq \exp \left(-\int_{0}^{r} h^{-1}(u) d u\right), \quad r \in\left(0, h\left(s_{0}-\right)\right) .
$$

Proof: If $\int \rho(K) \Lambda(d K)=0$, then $\rho(Z) \equiv 0$ a.s. and the result is trivial. Hence we can assume that $\int \rho(K) \Lambda(d K)>0$. We next show that then $\nu^{*}(0, \infty)>0$. By definition of $\nu^{*}$ it is sufficient to show for each $K \in \mathcal{F}^{\prime}$ that $\rho^{*}(K)>0$ whenever $\rho(K)>0$. Since $\tau>0$ on $[0, \infty)$, it is sufficient to prove that $\Lambda\left(\mathcal{F}_{y}^{\prime}\right)<\infty$ for $\rho$-a.e. $y \in K$. But this follows from

$$
\int_{K} \Lambda\left(\mathcal{F}_{y}^{\prime}\right) \rho(d y)=\iint \mathbf{1}\{y \in L\} \mathbf{1}\{y \in K\} \Lambda(d L) \rho(d y)=\int \rho(K \cap L) \Lambda(d L),
$$

which is finite by (3.1).

Since $\nu^{*}(0, \infty)>0$ we obtain for each $s \in\left(0, s_{0}\right)$ that

$$
\frac{d}{d s} \int \phi(s u) \nu^{*}(d u)=h(s) \in(0, \infty), \quad \frac{d}{d s} h(s)=\int u^{2} e^{s u} \nu^{*}(d u) \in(0, \infty) .
$$

In view of Theorem 3.4 the proof can now be finished as that of Houdré (2002, Theorem 1). 
Remark 3.7. Proposition 3.2 in $\mathrm{Wu}(2000)$ implies (3.7) with $\nu$ instead of $\nu^{*}$. Since $\nu^{*} \leq \nu$, our result improves this inequality. The larger $y \mapsto \Lambda\left(\mathcal{F}_{y}^{\prime}\right)$ the larger the improvement. Recall from (3.4) that $\mathbb{P}(y \in Z)=1-\exp \left(-\Lambda\left(\mathcal{F}_{y}^{\prime}\right)\right)$ is the probability that the point $y \in \mathbb{Y}$ is covered by $Z$. Our concentration inequality takes into account these covering probabilities and hence the overlapping of distinct grains.

In the sequel we use the (decreasing) function $\psi:[0, \infty) \rightarrow(-\infty, \infty]$, defined by

$$
\psi(z):=1-\frac{1}{z}(1+z) \log (1+z), \quad z>0,
$$

and $\psi(0):=\infty$. We also define

$$
m_{i}:=\int_{0}^{\infty} u^{i} \nu^{*}(d u), \quad i \in\{0,1,2\} .
$$

The proof of the following corollary of Theorem 3.6 is similiar to that of Houdré (2002, Corollary 1).

Corollary 3.8. Assume that (3.1) holds and that $\nu^{*} \neq 0$. Assume also that there is some $a>0$ such that $\rho(K) \leq a$ for $\Lambda$-a.e. $K \in \mathcal{F}^{\prime}$. Then we have for each $i \in\{0,1,2\}$ that

$$
\mathbb{P}(\rho(Z)-\mathbb{E}[\rho(Z)] \geq r) \leq \exp \left[\frac{r}{a} \psi\left(\frac{a^{i-1} r}{m_{i}}\right)\right], \quad r>0 .
$$

Proof: We first note that $h\left(s_{0}-\right)=\infty$. This follows by $\nu^{*} \neq 0$ once we have shown that $s_{0}=\infty$. To this end, let $s>0$. Then, we have $\int \mathbf{1}\{\rho(K)>1\} e^{s \rho(K)} \Lambda(d K) \leq$ $e^{s a} \int \rho(K) \Lambda(d K)$ which is finite by (3.1).

Let $i \in\{0,1,2\}$. In the case $i=0$ we can assume that $m_{0}=\nu^{*}([0, \infty))>0$. (Otherwise there is nothing to prove.) It is easy to see that $h(s) \leq m_{i} a^{1-i}\left(e^{s a}-1\right)$ for all $s>0$; cf. the proof of Houdré (2002, Corollary 1$)$ for the case $i=2$. Therefore

$$
h^{-1}(r) \geq \frac{1}{a} \log \left(1+\frac{r a^{i-1}}{m_{i}}\right), \quad r>0 .
$$

Since

$$
-\int_{0}^{r} \log (1+z u) d u=r \psi(z r), \quad r>0,
$$

for each $z>0$, we deduce the assertion from Theorem 3.6.

Example 3.9. In this example we specialize the setting of this section to the case $\mathbb{Y}=\mathbb{R}^{d}$ for some fixed integer $d \in \mathbb{N}$. We set $\mathcal{F}:=\mathcal{F}\left(\mathbb{R}^{d}\right)$. We fix $r \in[0, d]$ and let $\lambda_{r}$ denote the $r$-dimensional Hausdorff measure on $\mathbb{R}^{d}$. Let $\mathcal{F}^{r}$ denote the set of all $C \in \mathcal{F}$ such that $\lambda_{r}(C \cap \cdot)$ is a locally finite measure on $\mathbb{R}^{d}$. By Zähle (1982, Corollary 2.1.5), this is a measurable set, that is $\mathcal{F}^{r} \in \mathcal{B}(\mathcal{F})$. Let $W \subset \mathbb{R}^{d}$ be a compact set and define $\rho:=\lambda_{r}(W \cap \cdot)$. By Zähle (1982, Theorem 2.1.3), we have that $K \mapsto \rho(K)$ is measurable on $\mathcal{F}^{r}$, so that the pair $\left(\mathcal{F}^{r}, \rho\right)$ satisfies the general assumptions of this section (with $\mathcal{F}^{\prime}=\mathcal{F}^{r}$ ). 


\section{Stationary Boolean models}

In this section we consider the setting of Example 3.9 in the case $r=d$. We let $\eta$ be a Poisson process on the space $\mathcal{F}^{d}$ of all closed sets $K \subset \mathbb{R}^{d}$ with $\lambda_{d}(K)<\infty$. We assume here that the intensity measure $\Lambda$ of $\eta$ is of the translation invariant form

$$
\Lambda=\int_{\mathcal{F}^{d}} \int_{\mathbb{R}^{d}} 1\{K+x \in \cdot\} d x \mathbb{Q}(d K),
$$

where $K+x:=\{y+x: y \in K\}$ and $\mathbb{Q}$ is a $\sigma$-finite measure on $\mathcal{F}^{d}$ satisfying

$$
0<\gamma_{1}:=\int \lambda_{d}(K) \mathbb{Q}(d K)<\infty
$$

Example 4.1. Let $\mathbb{Q}_{0}$ be a probability measure on $\mathcal{F}^{d}$ satisfying $\int \lambda_{d}(K) \mathbb{Q}_{0}(d K)<$ $\infty$ and let $\rho_{0}$ be a measure on $(0, \infty)$ such that $\int_{0}^{\infty} r^{d} \rho_{0}(d r)<\infty$. Assume that

$$
\mathbb{Q}=\iint \mathbf{1}\{r K \in \cdot\} \rho_{0}(d r) \mathbb{Q}_{0}(d K) .
$$

Then

$$
\begin{aligned}
\int \lambda_{d}(K) \mathbb{Q}(d K) & =\iint \lambda_{d}(r K) \rho_{0}(d r) \mathbb{Q}_{0}(d K) \\
& =\int r^{d} \rho_{0}(d r) \int \lambda_{d}(K) \mathbb{Q}_{0}(d K)<\infty .
\end{aligned}
$$

As in Section 3 we define $Z:=Z(\eta)$, where $Z(\mu), \mu \in \mathbf{N}$, is given by (3.2) and the $\sigma$-finiteness of $\Lambda$ will be checked below. We fix a closed set $W \subset \mathbb{R}^{d}$ with positive finite volume and derive concentration inequalities for the Poisson functional

$$
F:=\lambda_{d}(Z \cap W) .
$$

We do this by applying the results of the previous section in the case $\rho:=\lambda_{d}(W \cap \cdot)$. Let $p:=1-e^{-\gamma_{1}}$. By (3.4), we have $p=\mathbb{P}(0 \in Z)$. Moreover, Fubini's theorem and (4.3) below imply that $\mathbb{E}[F]=p \lambda_{d}(W)$, so that $p$ is the volume fraction of $Z$; see also Last and Penrose (2017, (17.8)).

Theorem 4.2. Assume that (4.2) holds. Then the Poisson functional $F=\lambda_{d}(Z \cap$ W) satisfies

$\mathbb{P}(F-\mathbb{E}[F] \geq r) \leq \exp \left(\inf _{s>0}\left(\frac{p}{\gamma_{1}} \iint \phi\left(s \lambda_{d}((K+x) \cap W)\right) d x \mathbb{Q}(d K)-s r\right)\right), \quad r>0$.

Proof: We wish to apply Theorem 3.4 in the case $\rho=\lambda_{d}(W \cap \cdot)$.

Set $\mathbb{Q}^{\prime}(d K):=\mathbf{1}\left\{\lambda_{d}(K)>0\right\} \mathbb{Q}(d K)$ and $\Lambda^{\prime}(d K):=\mathbf{1}\left\{\lambda_{d}(K)>0\right\} \Lambda(d K)$ and let $\eta^{\prime}$ be a Poisson process with intensity measure $\Lambda^{\prime}$. Then $\lambda_{d}\left(Z\left(\eta^{\prime}\right) \cap W\right)$ has the same distribution as $\lambda_{d}(Z(\eta) \cap W)$. Hence we can assume without loss of generality that $\lambda_{d}(K)>0$ for $\mathbb{Q}$-a.e. $K$. In particular $\Lambda$ is then $\sigma$-finite.

For each Borel set $K \subset \mathbb{R}^{d}$ we have that

$$
\begin{aligned}
\int \lambda_{d}(W \cap(K+x)) d x & =\iint 1\{y \in K+x\} 1\{y \in W\} d y d x \\
& =\iint 1\{y \in K\} 1\{y+x \in W\} d y d x
\end{aligned}
$$


By Fubini's theorem and (4.1) we obtain that

$$
\int \lambda_{d}(W \cap K) \Lambda(d K)=\lambda_{d}(W) \int \lambda_{d}(K) \mathbb{Q}(d K),
$$

so that (4.2) implies assumption (3.1).

In what follows we often use that $\int \lambda_{d}((K+x) \cap L) d x=\lambda_{d}(K) \lambda_{d}(L)$ for all Borel sets $K, L \subset \mathbb{R}^{d}$, a direct consequence of Fubini's theorem. Let $s>0$. Then

$$
\begin{gathered}
\iint 1\left\{\lambda_{d}((K+x) \cap W)>1\right\} e^{s \lambda_{d}((K+x) \cap W)} d x \mathbb{Q}(d K) \\
\leq e^{s \lambda_{d}(W)} \iint \lambda_{d}((K+x) \cap W) d x \mathbb{Q}(d K) \\
=e^{s \lambda_{d}(W)} \lambda_{d}(W) \int \lambda_{d}(K) \mathbb{Q}(d K)<\infty
\end{gathered}
$$

Therefore we have $s_{0}=\infty$, where $s_{0}$ is given by (3.6).

As at (3.3) we define $\mathcal{F}_{x}^{d}:=\left\{K \in \mathcal{F}^{d}: x \in K\right\}$ for $x \in \mathbb{R}^{d}$. From (4.1) we obtain that

$$
\Lambda\left(\mathcal{F}_{x}^{d}\right)=\Lambda\left(\mathcal{F}_{0}^{d}\right)=\int \lambda_{d}(K) \mathbb{Q}(d K)=\gamma_{1}
$$

Hence $\tau\left(\Lambda\left(\mathcal{F}_{x}^{d}\right)\right)=p / \gamma_{1}$. Therefore the measure $\nu^{*}$ defined by (3.5) is given by

$$
\nu^{*}=\frac{p}{\gamma_{1}} \int \mathbf{1}\left\{\lambda_{d}(K \cap W) \in \cdot\right\} \Lambda(d K) .
$$

Hence Theorem 3.4 implies the assertion.

The concentration inequality of Theorem 4.2 is rather complicated. In the sequel we shall derive more explicit versions. We use the function $\psi$ defined by (3.13) and the constant

$$
\gamma_{2}:=\int \lambda_{d}(K)^{2} \mathbb{Q}(d K)
$$

Corollary 4.3. Assume that (4.2) holds and that $a>0$ is such that $\lambda_{d}(K \cap W) \leq a$ for $\Lambda$-a.e. $K \in \mathcal{F}^{d}$. Then $F=\lambda_{d}(Z \cap W)$ satisfies

$$
\begin{aligned}
& \mathbb{P}(F-\mathbb{E}[F] \geq r) \leq \exp \left[\frac{r}{a} \psi\left(\frac{r}{p \lambda_{d}(W)}\right)\right], \quad r>0, \\
& \mathbb{P}(F-\mathbb{E}[F] \geq r) \leq \exp \left[\frac{r}{a} \psi\left(\frac{a \gamma_{1} r}{p \lambda_{d}(W) \gamma_{2}}\right)\right], \quad r>0 .
\end{aligned}
$$

Proof: We can apply Corollary 3.8. Using (4.4) and (4.1) we obtain that

$$
m_{1}=\frac{p}{\gamma_{1}} \iint \lambda_{d}((K+x) \cap W) d x \mathbb{Q}(d K)=p \lambda_{d}(W) .
$$

Inequality (4.5) now follows from the case $i=1$ of Corollary 3.8.

Similarly we obtain that

$$
\begin{aligned}
m_{2} & =\frac{p}{\gamma_{1}} \iint \lambda_{d}((K+x) \cap W)^{2} d x \mathbb{Q}(d K) \\
& \leq \frac{p}{\gamma_{1}} \int \lambda_{d}(K) \int \lambda_{d}((K+x) \cap W) d x \mathbb{Q}(d K)=\frac{p}{\gamma_{1}} \lambda_{d}(W) \int \lambda_{d}(K)^{2} \mathbb{Q}(d K) .
\end{aligned}
$$

Since $\psi$ is decreasing, the inequality (4.6) follows from the case $i=2$ of Corollary 3.8. 
Remark 4.4. Suppose there exists $a>0$ such that $\lambda_{d}(K) \leq a$ for $\mathbb{Q}$-a.e. $K \in \mathcal{F}^{d}$. Then (4.6) is better than (4.5). Indeed, since $\psi$ is decreasing the right-hand side of (4.6) is smaller than that of (4.5) iff $a \gamma_{1} / \gamma_{2} \geq 1$. If there exist $\gamma>0$ and $K_{0} \in \mathcal{F}^{d}$ with $\lambda_{d}\left(K_{0}\right)>0$ such that $\mathbb{Q}=\gamma \delta_{K_{0}}$, then both inequalities yield

$$
\mathbb{P}(F-\mathbb{E}[F] \geq r) \leq \exp \left[\frac{r}{\lambda_{d}\left(K_{0}\right)} \psi\left(\frac{r}{p \lambda_{d}(W)}\right)\right], \quad r>0 .
$$

Remark 4.5. The bound (4.7) is quite sharp. Indeed, if $X$ is a Poisson distributed random variable then $\mathbb{P}(X-\mathbb{E}[X] \geq r) \sim(2 \pi(\mathbb{E}[X]+r))^{-1 / 2} \exp (r \cdot \psi(r / \mathbb{E}[X]))$, as $r \rightarrow \infty$, see p. 1225 in Houdré (2002).

Remark 4.6. Choosing $a=\lambda_{d}(W)$ in (4.5) yields

$$
\mathbb{P}(F-\mathbb{E}[F] \geq r) \leq \exp \left[\frac{r}{\lambda_{d}(W)} \psi\left(\frac{r}{p \lambda_{d}(W)}\right)\right], \quad r>0 .
$$

The advantage of this result is that it holds under the only assumption (4.2). The disadvantage is the occurrence of $\lambda_{d}(W)^{-1}$ as a factor of $r$ outside the logarithmic term. This is in contrast with the situation in Remark 4.4.

If $\lambda_{d}(\cdot)$ is not essentially bounded w.r.t. $\mathbb{Q}$, we need an exponential moment assumption on $\mathbb{Q}$ to improve (4.8) at least partially. Define a function $\tilde{h}:[0, \infty) \rightarrow$ $[0, \infty]$ by

$$
\tilde{h}(s):=\int \lambda_{d}(K)\left(e^{s \lambda_{d}(K)}-1\right) \mathbb{Q}(d K), \quad s \geq 0 .
$$

Corollary 4.7. Assume that (4.2) holds. Then the Poisson functional $F=\lambda_{d}(Z \cap$ $W)$ satisfies

$$
\mathbb{P}(F-\mathbb{E}[F] \geq r) \leq \exp \left(-\int_{0}^{r} \tilde{h}^{-1}\left(\frac{\gamma_{1} u}{p \lambda_{d}(W)}\right) d u\right), \quad r>0 .
$$

Proof: We wish to apply Theorem 3.6. Recall the definition (3.12) of the function $h$. By (4.4) we have for each $s \geq 0$ that

$$
\begin{aligned}
h(s) & =\frac{p}{\gamma_{1}} \iint \lambda_{d}((K+x) \cap W)\left(e^{s \lambda_{d}((K+x) \cap W)}-1\right) d x \mathbb{Q}(d K) \\
& \leq \frac{p}{\gamma_{1}} \iint \lambda_{d}((K+x) \cap W)\left(e^{s \lambda_{d}(K)}-1\right) d x \mathbb{Q}(d K)=\frac{p \lambda_{d}(W)}{\gamma_{1}} \tilde{h}(s) .
\end{aligned}
$$

Hence we have for each $r>0$ that $h^{-1}(r) \geq \tilde{h}^{-1}\left(\gamma_{1} r /\left(p \lambda_{d}(W)\right)\right)$, so that Theorem 3.6 and the identity $s_{0}=\infty$ imply the assertion once we have shown that $\lim _{s \rightarrow \infty} h(s)=\infty$. But this follows from $\nu^{*}((0, \infty))>0$ (a consequence of $\gamma_{1}>$ $0)$.

We illustrate Corollary 4.7 with two examples. Let $c:=\gamma_{1} /\left(p \lambda_{d}(W)\right)$.

Example 4.8. Assume that $\mathbb{Q}\left(\left\{K: \lambda_{d}(K) \in d u\right\}\right) \leq \alpha u^{-1} e^{-\beta u} d u$, where $\alpha, \beta>0$. On the right-hand side we have here the Lévy measure of the gamma distribution with shape parameter $\alpha>0$ and rate parameter $\beta>0$; see e.g. Last and Penrose (2017, Example 15.6). For instance, this assumption is satisfied with $\alpha=1 / d$ if $\mathbb{Q} \leq \int \mathbf{1}\left\{r K_{0} \in \cdot\right\} r^{-1} e^{-\beta r^{d}} d r$, where $K_{0} \in \mathcal{F}^{d}$ has $\lambda_{d}\left(K_{0}\right)=1$. Let $s \in(0, \beta)$. A simple calculation shows that $\tilde{h}(s) \leq \alpha /(\beta-s)-\alpha / \beta$, so that

$$
\tilde{h}^{-1}(r) \geq \beta-\frac{\alpha \beta}{\beta r+\alpha}, \quad r>0 .
$$


It follows that

$$
\int_{0}^{r} \tilde{h}^{-1}(u) d u \geq \beta r-\alpha \log \left(1+\frac{\beta r}{\alpha}\right), \quad r>0 .
$$

Therefore we obtain from Corollary 4.7

$$
\mathbb{P}(F-\mathbb{E}[F] \geq r) \leq \exp \left(-\beta r+\frac{\alpha}{c} \log \left(1+\frac{\beta c r}{\alpha}\right)\right) .
$$

Example 4.9. Assume that $\mathbb{Q}\left(\left\{K: \lambda_{d}(K) \in d u\right\}\right) \leq \beta^{\alpha} / \Gamma(\alpha) u^{\alpha-1} e^{-\beta u} d u$, where $\alpha, \beta>0$. On the right-hand side we have here the gamma distribution with shape parameter $\alpha$ and scale parameter $\beta$. A similar calculation as in Example 4.8, yields $\tilde{h}(s) \leq \alpha \beta^{\alpha} /(\beta-s)^{\alpha+1}-\alpha / \beta$ for $s \in(0, \beta)$, so that

$$
\tilde{h}^{-1}(r) \geq \beta-\left(\frac{\alpha \beta^{\alpha+1}}{\beta r+\alpha}\right)^{1 /(\alpha+1)}, \quad r>0,
$$

and finally, by Corollary 4.7,

$$
\mathbb{P}(F-\mathbb{E}[F] \geq r) \leq \exp \left(-\beta r+\frac{\alpha+1}{c}\left(\left(\frac{\alpha+\beta c r}{\alpha}\right)^{\alpha /(\alpha+1)}-1\right)\right)
$$

Remark 4.10. Examples 4.8 and 4.9 are geometrically quite different. Assume in the second example that $\mathbb{Q}\left(\left\{K: \lambda_{d}(K) \in d u\right\}\right)=\beta^{\alpha} / \Gamma(\alpha) u^{\alpha-1} e^{-\beta u} d u$. Then each bounded set contains a finite number of grain centers (at least under a weak regularity assumption on $\mathbb{Q}$ ). Ignoring overlapping, each grain contributes a gamma distributed volume. Assume in the first example that $\mathbb{Q}\left(\left\{K: \lambda_{d}(K) \in d u\right\}\right)=$ $u^{-1} e^{-\beta u} d u$. Then each measurable set $B \subset \mathbb{R}^{d}$ with $0<\lambda_{d}(B)<\infty$ contains infinitely many grain centers. However, the sum of the volumes of balls centered in $B$ follows a gamma distribution with scale parameter $\beta$ and shape parameter $\lambda_{d}(B)$; see Last and Penrose (2017, Example 15.6). Roughly speaking, $Z \cap W$ might be interpreted as a finite union of random sets whose volumes are approximately gamma distributed. This might explain that the leading terms in both concentration inequalities are the same.

Our bounds of Corollary 4.3 improve significantly Theorem 3 in Heinrich (2005) which deals with the stationary Boolean model in $\mathbb{R}^{d}$ and which assumes $\mathbb{Q}$ to be a probability measure. The tail bound in Heinrich (2005) is only of order exp $(-\mathcal{O}(r))$ and therefore not able to reproduce the tail behaviour of the Poisson distribution in the special setting of Remark 4.4. Further, the constants we use arise naturally from the model and are much less involved than the ones in Heinrich (2005). Moreover, we do not require that the moment generating function of $\lambda_{d}\left(Z_{0}\right)$ exists but only make the milder moment assumptions $\gamma_{1}<\infty$, respectively $\gamma_{2}<\infty$.

We note that the general concentration inequalities derived in Bachmann and Peccati (2016) can be applied to some configurations of the stationary Boolean model in $\mathbb{R}^{d}$, too. At least in the case of bounded grains, this application already improves the corresponding result of Heinrich (2005). However, the functionals considered in Bachmann and Peccati (2016) appear unable to incorporate the volume fraction. To be more precise, in the setting of Corollary 4.3, the bound (4.6) is sharper than the inequality

$$
\mathbb{P}(F-\mathbb{E}[F] \geq r) \leq \exp \left(\frac{r}{a} \cdot \psi\left(\frac{a r}{\gamma_{2} \lambda_{d}(W)}\right)\right), \quad r \geq 0,
$$


obtained from Corollary 3.3 in Bachmann and Peccati (2016) with the bound

$$
\left.\int\left(D_{K} F\right)^{2} \Lambda(d K) \leq \iint \lambda_{d}(K+x) \cap W\right)^{2} d x \mathbb{Q}(d K) \leq \lambda_{d}(W) \gamma_{2} .
$$

Finally, we apply Theorem 2.5.

Proposition 4.11. Assume that (4.2) holds. Then

$$
\mathbb{P}[F-\mathbb{E}[F] \geq r] \leq \exp \left(-\frac{\gamma_{1}}{p \lambda_{d}(W)}\left(r+\mathbb{E}[F] \log \left(\frac{\mathbb{E}[F]}{r+\mathbb{E}[F]}\right)\right)\right), \quad r>0 .
$$

Proof: By Lemma 3.3 and equation (4.3), we have, for $\Lambda$-a.e. $K \in \mathcal{F}^{d}$,

$$
\int_{0}^{1} \int_{\mathbf{N}} D_{K} f\left(\eta_{t}+\mu\right) \Pi_{(1-t) \Lambda}(d \mu) d t \leq \rho(K \cap W) \frac{p}{\gamma_{1}} \leq \lambda_{d}(W) \frac{p}{\gamma_{1}} .
$$

Using the properness of $\eta$, we also get the bound

$$
\int D_{K} f\left(\eta-\delta_{K}\right) \eta(d K)=\sum_{K \in \eta} D_{K} f\left(\eta-\delta_{K}\right)=\sum_{K \in \eta} \lambda_{d}\left((K \cap W) \backslash \bigcup_{L \in \eta-\delta_{K}} L\right) \leq F .
$$

The assertion follows from Theorem 2.5 by truncation as in the proof of Theorem 3.4.

We note that $\int D_{K} f\left(\eta-\delta_{K}\right) \eta(d K)$ equals the volume of the set of points which are covered by exactly one grain. Thus, as the Mecke formula allows us to employ the functional $\int D_{K} f\left(\eta-\delta_{K}\right) \eta(d K)$, we are equipped with a finer tool to respect the interplay between the grains of $Z$.

Example 4.12. Let $\mathbb{Q}\left(\left\{K: \lambda_{d}(K) \in d u\right\}\right)=\beta e^{-\beta u} d u$, that is the volume of the typical grain is exponentially distributed. The larger $\beta$ (and therefore the smaller $p$ ) the better is the specific bound (4.10) in comparison to the general bound (4.11). If $\beta>0.14$, i.e. $p<0.9992$, then (4.10) outplays (4.11) uniformly. If $\beta<0.13$, it is the other way round. Between, (4.11) might be better only for small values of $r$. Comparing the more general bound (4.8) with (4.11), we observe the same behaviour. The latter wins when $p$ is large.

Acknowledgment: We wish to thank S. Bachmann and G. Peccati for discussing with us an early version of their paper Bachmann and Peccati (2016). We also thank two anonymous referees for several helpful comments. This work was supported by the German Research Foundation (DFG) through the research unit "Geometry and Physics of Spatial Random System" under the grant LA 965/6-2.

\section{References}

S. Bachmann and G. Peccati. Concentration bounds for geometric Poisson functionals: logarithmic Sobolev inequalities revisited. Electron. J. Probab. 21, Paper No. 6, 44 (2016). MR3485348.

S. Boucheron, G. Lugosi and P. Massart. Concentration inequalities using the entropy method. Ann. Probab. 31 (3), 1583-1614 (2003). MR1989444.

J.-C. Breton, C. Houdré and N. Privault. Dimension free and infinite variance tail estimates on Poisson space. Acta Appl. Math. 95 (3), 151-203 (2007). MR2317609. 
L. H. Y. Chen. Poincaré-type inequalities via stochastic integrals. Z. Wahrsch. Verw. Gebiete 69 (2), 251-277 (1985). MR779459.

H. Chernoff. A measure of asymptotic efficiency for tests of a hypothesis based on the sum of observations. Ann. Math. Statistics 23, 493-507 (1952). MR0057518.

S. N. Chiu, D. Stoyan, W. S. Kendall and J. Mecke. Stochastic geometry and its applications. Wiley Series in Probability and Statistics. John Wiley \& Sons, Ltd., Chichester, third edition (2013). ISBN 978-0-470-66481-0. MR3236788.

L. Heinrich. Large deviations of the empirical volume fraction for stationary Poisson grain models. Ann. Appl. Probab. 15 (1A), 392-420 (2005). MR2115047.

C. Houdré. Remarks on deviation inequalities for functions of infinitely divisible random vectors. Ann. Probab. 30 (3), 1223-1237 (2002). MR1920106.

C. Houdré, P. Marchal and P. Reynaud-Bouret. Concentration for norms of infinitely divisible vectors with independent components. Bernoulli 14 (4), 926-948 (2008). MR2543580.

C. Houdré and V. Pérez-Abreu. Covariance identities and inequalities for functionals on Wiener and Poisson spaces. Ann. Probab. 23 (1), 400-419 (1995). MR1330776.

C. Houdré and N. Privault. Concentration and deviation inequalities in infinite dimensions via covariance representations. Bernoulli 8 (6), 697-720 (2002). MR1962538.

D. Hug, G. Last and M. Schulte. Second-order properties and central limit theorems for geometric functionals of Boolean models. Ann. Appl. Probab. 26 (1), 73-135 (2016). MR3449314.

O. Kallenberg. Foundations of modern probability. Probability and its Applications (New York). Springer-Verlag, New York, second edition (2002). ISBN 0-38795313-2. MR1876169.

G. Last and M. Penrose. Lectures on the Poisson Process. Institute of Mathematical Statistics Textbooks. Cambridge University Press (2017). DOI: $10.1017 / 9781316104477$.

P. Massart. About the constants in Talagrand's concentration inequalities for empirical processes. Ann. Probab. 28 (2), 863-884 (2000). MR1782276.

G. Matheron. Random sets and integral geometry. John Wiley \& Sons, New YorkLondon-Sydney (1975). MR0385969.

N. Privault. Stochastic analysis in discrete and continuous settings with normal martingales, volume 1982 of Lecture Notes in Mathematics. Springer-Verlag, Berlin (2009). ISBN 978-3-642-02379-8. MR2531026.

R. Schneider and W. Weil. Stochastic and integral geometry. Probability and its Applications (New York). Springer-Verlag, Berlin (2008). ISBN 978-3-540-788584. MR2455326.

L. Wu. A new modified logarithmic Sobolev inequality for Poisson point processes and several applications. Probab. Theory Related Fields 118 (3), 427-438 (2000). MR1800540.

M. Zähle. Random processes of Hausdorff rectifiable closed sets. Math. Nachr. 108, 49-72 (1982). MR695116.

U. Zähle. Random fractals generated by random cutouts. Math. Nachr. 116, 27-52 (1984). MR762590. 